Europhys. Lett., 2 (10), pp. 739-745 (1986)

\title{
Phase Transitions in Two-Dimensional Kauffman Cellular Automata.
}

\author{
B. DeRrida and D. Stauffer $(*)$ \\ Service de Physique Théorique, CEN Saclay - F-91191 Gif-sur-Yvette, France
}

(received 21 May 1986; accepted in final form 30 June 1986)

PACS. 05.40. - Fluctuation phenomena, random processes, and Brownian motion.

PACS. 05.50. - Lattice theory and statistics: Ising problems.

\begin{abstract}
In the random Boolean networks suggested by Kauffman, each site is changed to random rules depending on neighbours of this site. One can define two kinds of models, the annealed for which the random rules are changed at each time step, and the quenched for which the random rules remain fixed. We consider this model mostly on a square lattice with nearestneighbour interactions. In the annealed case the phase transition observed in the overlap of configurations differing in the initial conditions is related to directed percolation on the bodycentred cubic lattice; in the quenched case, the original Kauffman model, the phase transition is seen in the variation of this final overlap with the initial overlap.
\end{abstract}

In the field of cellular automata [1], the Kauffman model [2] is one of the most random ones. Each of $N$ lattice sites contains a Boolean variable $\sigma$ (or spin) which is either zero or unity. The time evolution of this model is determined by $N$ functions $f_{i}$, one chosen for each site independently, and by the choice of $K$ input sites $j_{1}(i), j_{2}(i), \ldots, j_{K}(i)$ for each site $i$. Thus the value $\sigma_{i}$ at site $i$ for time $t+1$ is given by

$$
\sigma_{i}(t+1)=f_{i}\left(\sigma_{j_{1}}(t), \sigma_{j_{2}}(t), \ldots, \sigma_{j_{K}}(t)\right) \quad(i=1,2, \ldots, N) .
$$

Each Boolean function $f_{i}$ is specified, once its value is given for each of the $2^{K}$ possible configurations of its arguments.

Many previous studies [2-4] investigated the situation originally suggested by KAUFFMAN [2] and which we call here the «infinite dimension» limit. The input sites $j_{1}(i), j_{2}(i), \ldots, j_{K}(i)$ were chosen randomly among the $N$ lattice sites.

Also for each site, the value of each function $f_{i}$ was 1 with probability $p=\frac{1}{2}$ and 0 with probability $1-p=\frac{1}{2}$. This parameter $p$ is related to Walker's "internal homogeneity" [2]. We call this case of infinite range of interactions the infinite dimension limit, since on

(*) LHMP, ESPCI, 10 rue Vauquelin, F-75231 Paris Cedex 05; permanently at Institute of Theoretical Physics, Cologne University, D-5000 Koln 41, West Germany. 
average the value of a spin at time $t$ depends on the values of an exponentially increasing number, like $K^{t}$, of spins at $t=0$. In this infinite-dimension case, ref. [3] compared two models: The quenched model, for which the functions $f_{i}$ and the input sites $j_{1}(i), j_{2}(i), \ldots, j_{K}(i)$ were chosen randomly at time $t=0$ and remain fixed at all later times; and the annealed model for which a new function $f_{i}$ and, for infinite dimensions, also new input sites were chosen randomly at each time step and which is thus more similar to other probabilistic cellular automata [6].

These two models are a priori very different: for example the quenched model has limit cycles, which are absent in the annealed model. However [3], in the thermodynamic limit $N \rightarrow \infty$ some quantities were shown to be the same in the two models for infinite dimension. Defining the overlap

$$
a_{12}(t)=N^{-1} \sum_{i=1}^{N}\left\{1-\left(\sigma_{i}(t)-\rho_{i}(t)\right)^{2}\right\}=1-\psi(t),
$$

as the normalized number of spins which are the same in two different spin configurations $\left\{\sigma_{i}\right\}$ and $\left\{\hat{f}_{i}\right\}$ for the same set of functions $\left\{f_{i}\right\}$, or $\psi$ as the "distance» between these two configurations, this overlap was shown to be exactly the same in the quenched and the annealed model, for $N \rightarrow \infty$.

In both models, when a parameter is varied, e.g. the number $K$ of inputs, two possible phases can be observed. A first phase, corresponding to $K \leqslant 2$, which we now call the frozen phase, was characterized by distances $\psi$ vanishing for long times; i.e. for any initial overlap $a_{12}(t=0)$, one has

$$
\lim _{t \rightarrow \infty} a_{12}(t)=1
$$

In this frozen phase, two arbitrarily chosen spin configurations, iterated long enough according to the same rules $f_{i}$, will become identical after a sufficiently long time (more precisely, they will not differ by a macroscopic number of spins).

In the second phase, which we call chaotic, corresponding to $K>2$ in the infinitedimension case, for any initial $a_{12}(t=0) \neq 1$ one has

$$
\lim _{t \rightarrow \infty} a_{12}(t)=a_{12}^{*} \neq 1
$$

with the final overlap $a_{12}^{*}$ independent of the initial overlap $a_{12}(0) \neq 1$. Criteria (3) and (4) are simple consequences of the time evolution equation

$$
a_{12}(t+1)=\left\{1+\left(a_{12}(t)\right)^{K}\right\} / 2
$$

derived and studied in ref. [3]. It was shown [3] that as a parameter (like $K$ ) varied, a transition between a frozen and a chaotic phase occured $\left(K_{0}=2\right)$.

In the present work we investigate the properties of the same model in finite dimension, i.e. for finite interaction range. The spins $\sigma_{1}$ are located on a periodic lattice and depend in their time evolution on their $K$ nearest neighbours. For a chain in one dimension $K=2$, whereas $K=4$ on the square lattice. Even in the annealed model, these neighbour spins are always the same $K$ lattice neighbours, and thus the spins are spatially correlated, as customary in statistical mechanics. Now the spin value after $t$ iterations depends on a number $\propto t^{d}$ of spins at $t=0$, in contrast to the exponential increase for infinite dimensions.

The questions we would like to study are: Which properties of infinite dimension persist 
in finite dimension? For example,

i) Are annealed and quenched model still identical for $N \rightarrow \infty$ ?

ii) Are eqs. (3), (4) still valid?

iii) Are there still two phases, frozen and chaotic?

iv) If yes, is the phase transition related to other models of statistical physics?

v) Are there new effects in finite cimension which are absent in infinite dimension?

ATLAN et al. [2], FOGELMAN [4] and VICHNIAC et al. [5] already investigated numerically some possible phase transitions using different criteria, like whether a small initial perturbation of a limit cycle is spreading, using mostly two or three input spins (instead of our four); however, these authors did not work with our distance function $\psi(t)$ between two states.

For numerical investigations of possible phase transitions we prefer a continuously varying parameter. Thus instead of varying $K$ as in the analytic treatment of infinite dimension [3], we now keep the number $K$ of neighbours fixed and vary the probability $p$ that the random function $f_{i}$ is unity $[3,4]$

$$
f_{i}=1 \text { with probability } p, \quad f_{i}=0 \text { with probability } 1-p .
$$

Because of the symmetry between the Boolean values 0 and 1 , if initially half of the spins are zero, only the range $0 \leqslant p \leqslant \frac{1}{2}$ needs to be studied.

For infinite dimension, eq. (5) is easily generalized to this case

$$
a_{12}(t+1)=1-\left\{1-\left(a_{12}(t)\right)^{K}\right\} 2 p(1-p) .
$$

By looking at the stability of the fixed point $a_{12}^{*}=1$ corresponding to identical spin distributions, we see that again for $K \leqslant 2$ only the frozen phase persists, whereas for $K>2$ also the chaotic phase exists in the interval $p_{\mathrm{c}}<p<1-p_{\mathfrak{c}}$, with a critical value $p_{\mathrm{c}}$ given by $2 K p_{\mathrm{c}}\left(1-p_{\mathrm{c}}\right)=1$; for example $p_{\mathrm{c}}=0.1464$ for $K=4$. If $p$ approaches $p_{\mathrm{c}}, 1-a_{12}^{*}$ vanishes linearly in $p-p_{\mathrm{c}}$.

Let us first discuss the annealed model in finite dimension. First we show that, as far as the overalp between two configurations is concerned, the $d$-dimensional annealed model is equivalent to directed percolation [6] in $d+1$ dimensions, as was pointed out before [6] for different cellular automata. From a $d$-dimensional lattice $L$ one can build a $(d+1)$ dimensional lattice $L^{\prime}$ by adding the time direction. Each spin of the plane $t+1$ is connected to its $K$ neighbours (input sites) of the plane $t$. Thus a one-dimensional chain $L$ gives in this way a square lattice, whereas the Kaufman model on the square lattice, with the time dimension added, gives a body centred cubic lattice.

Let us now iterate according to the same rules $f_{i}$ two configurations $\left\{\sigma_{i}\right\}$ and $\left\{\rho_{i}\right\}$ on the same lattice $L$. We denote a site $(i, t)$ of the $(\sigma+1)$-dimensional lattice $L^{\prime \prime}$ as "occupied", if $\sigma_{i}(t)$ and $\sigma_{i}(t)$ differ, whereas we call it "empty", if they have the same value. A site $i$ at time $t+1$ would always be empty if its $K$ neighbours at time $t$ were empty. If at least one of the $K$ neighbours is occupied at time $t$, then the site will be occupied at time $t+1$ with probability $2 p(1-p)$, since this is the probability that its function $f_{i}$ gives different values from the two different neighbour configurations. Thus the distances $\psi$ (or overlaps $a_{12}$ ) in the $d$ dimensional annealed Kaufman model on lattice $L$ reduce to counting the number of sites in the infinite cluster of the directed site percolation problem [6] in $d+1$ dimensions on the 
expanded lattice $L^{\prime}$, with a concentration

$$
x=2 p(1-p)
$$

of occupied sites. (For the overlap at the new site it does not matter if all the four neighbours are different, or only one, two or three; in all these cases this site becomes occupied with the probability $x$ of eq. (8). Thus we deal with site percolation, not with bond percolation.) If $P_{\infty}$ is the probability that a site belongs to the infinite cluster of the percolation problem on $L^{\prime}$, then for long times we have a fixed point

$$
1-a_{12}^{*}=\psi(t=\infty)=P_{\infty}(x)
$$

for the distances between different configurations, independent of the initial distance $\psi(t=0)$ (provided it was not zero). In this sense, criteria (3), (4), derived initially [3] for infinite dimension only, remain valid also for finite dimensions. Moreover, percolation theory [6] now tells us how the limiting distance $\psi$ vanishes if $p$ approaches $p_{\mathrm{c}}$ from above. Our rough estimate of $p_{c}=0.22$, taken from the condition $\psi=0$ (see later in fig. 2 ), is in good agreement with the more accurate $x_{c}=0.344$ of directed percolation on the b.c.c. lattice, which gives $p_{\mathrm{c}}=0.221$.

For the chain with nearest-neighbour interactions, $K=2$, only the frozen phase exists, not the chaotic phase, because the concentration $x=2 p(1-p)$ varies between 0 and $\frac{1}{2}$ only, and thus cannot reach the square-lattice percolation threshold [6] $x_{c}=0.706$. However, by working with both nearest and next-nearest neigbours $(K=4)$, one can decrease $x_{\mathrm{c}}$ and then observes also a chaotic phase for $p$ larger than about $\frac{1}{4}$ in one dimension.

Thus in the annealed case the situation is qualitatively similar to infinite dimensions except for the numerical values of thresholds and critical exponents: Two different phases, characterized by criteria (3) and (4), are possible in all dimensions including $d=1$. We now shall see that the quenched $d$-dimensional model behaves qualitatively different, with the final distance $\psi(t \rightarrow \infty)$ in the chaotic phase depending on the initial distance $\psi(t=0)$.

Let us now discuss the quenched model. There, in contrast to the annealed case, we did not find a relation between the Kauffman automata and some already studied percolation model, and thus rely on computer simulations on the square lattice. Most of our algorithm was automatically vectorized on a Cray machine and investigated there more than one million sites per second, with two configurations following the same iteration rules. Lattices containing up to $250 \times 250$ sites were simulated, with ten different runs for each parameter set. Some simulations in one dimension with $K=4$ used 10000 sites. For $t=0$ half of the spins were 0 , the others were 1 . We iterated the system up to 1000 time steps. (To vary the initial overlap $a_{12}$, we first produced two identical configurations, and then overturned randomly selected spins in one of the two sets.) Of course, neither the time over which the sample was observed, nor the sample size, were truely infinite as would be required theoretically. (For $t \rightarrow \infty$, we see small oscillations (within a limit cycle) in the fraction of zero spins which diminish only slightly if we switch from size $50 \times 50$ to size $250 \times 250$. We also found $\psi(\infty)$ to vary slightly if the spin configurations were still chosen independently, but with more spins zero than unity.)

We observed in the quenched model a threshold concentration $p_{\mathrm{c}}$ near $\frac{1}{4}$. In contrast to the annealed case as well as the quenched model for infinite dimension, the final distance $\psi(t \rightarrow \infty)=1-a_{12}$ does not vanish for concentrations $p$ below the threshold $p_{c}$ and it does depend on the initial distance $\psi(t=0)$ between the states. Figure 1 shows $\psi(\infty)$ as function of $\psi(0)$ for the quenched model in two dimensions. No such variation with the initial overlap is observed in the annealed case. Moreover, there is a discontinuity in the variation with $\varphi(0)$ : 


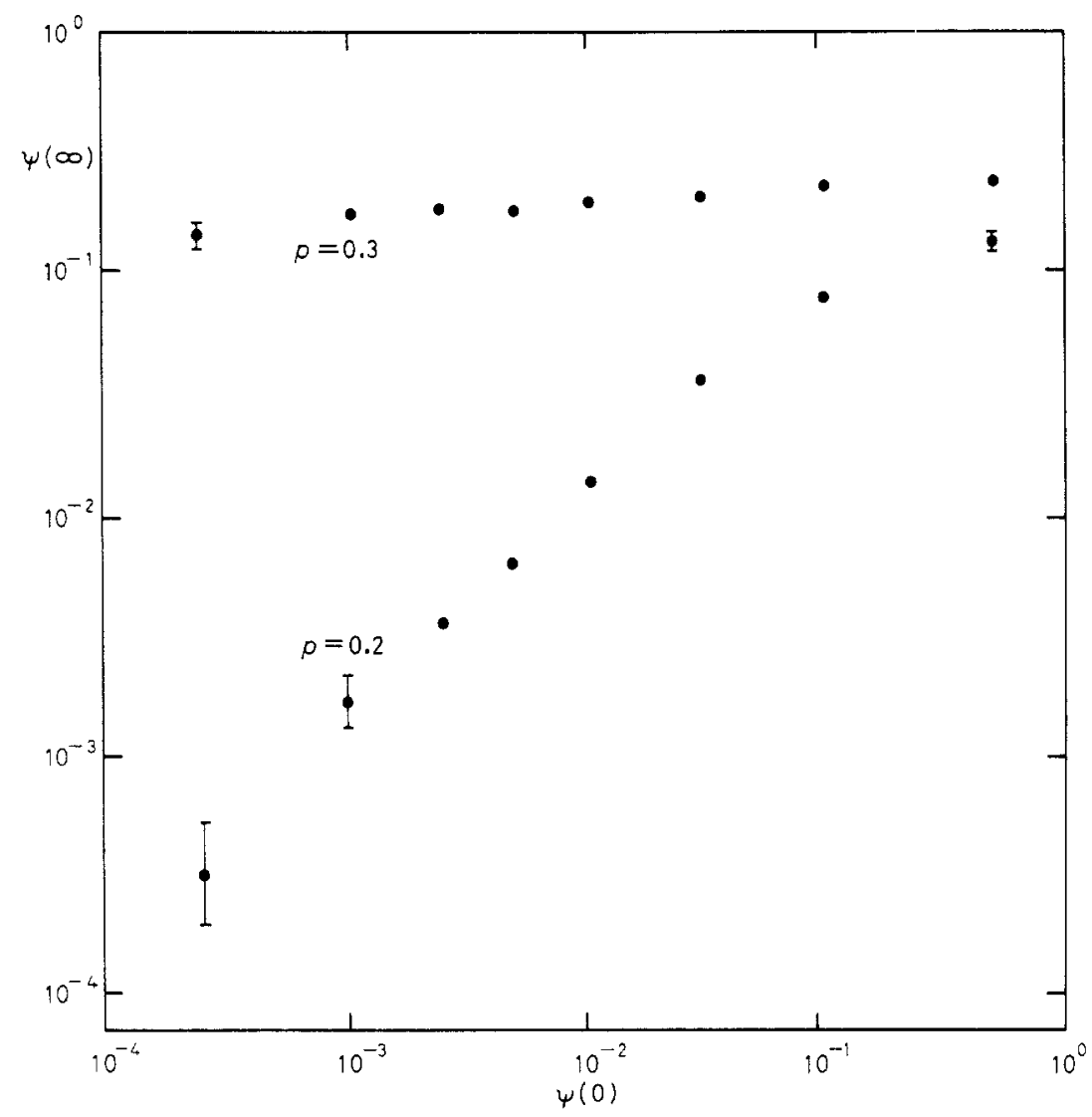

Fig. 1. - Log-log plot of final distance $\psi(\infty)$ vs. initial distance $\psi(0)$ at $p=0.3$ (chaotic phase) and $p=0.2$ (frozen phase). For $p=0.2$ we see that $\psi(\infty)$ is linear in $\psi(0)$ for small $\psi(0)$. In contrast, for $p=0.3, \psi(\infty)$ approaches a finite limit for $\psi(0) \rightarrow 0$. This difference indicates our phase transition.

If the initial distance is exactly zero, i.e. if the two states are identical, it always stays exactly at zero. If the initial distance $\psi(0)$ is very small, then for $p$ larger than about $\frac{1}{4}$ it increases drastically with time, until it reaches its asymptotic value $\psi(\infty)$; for small $p$, on the other hand, $\psi(t)$ stays quite small.

Thus within our numerical accuracy we see a discontinuity in $\psi(\infty)$ for $p$ above some threshold $p_{c}$, but not below:

$$
\begin{cases}\lim _{\psi(0) \rightarrow 0} \psi(t=\infty)=Q(p)>0, & \text { if } p>p_{\mathrm{c}}, \\ \lim _{\langle(0) \rightarrow 0} \psi(t=\infty)=0, & \text { if } p<p_{\mathrm{c}}, \\ \lim _{\langle(0) \rightarrow 0} \psi(\infty) / \psi(0)=\chi(p)>0, & \text { if } p<p_{\mathrm{c}}, \\ \psi(\infty)=0, & \text { if } \psi(0)=0, \text { for all } p .\end{cases}
$$

The discontinuity $Q(p)$ is seen in fig. 2, together with the asymptotic distance $\psi(t=\infty)$ estimated for both the quenched and the annealed model with the two initial states being uncorrelated $\left(\psi(0)=\frac{1}{2}\right)$. As usual in critical phenomena, close to the threshold $p_{\mathrm{c}}$ near 0.26 the transition is rounded due to finite $\psi(0)$, finite number of iterations, and finite system size. 


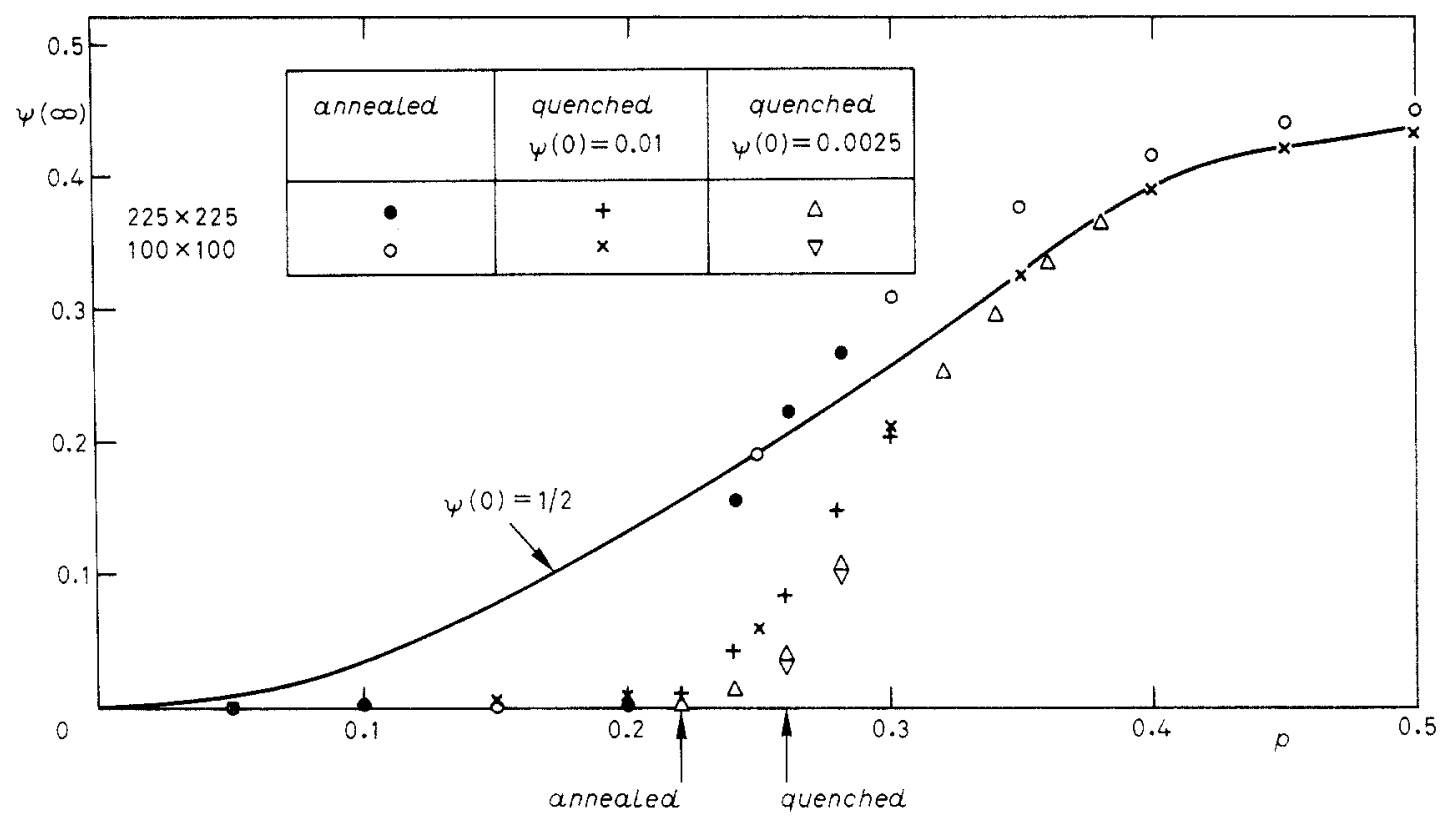

Fig. 2. - Variation of final distance $\psi(\infty)$ vs. $p$ for the annealed and the quenched model on the square lattice; points from simulations up to 1000 time steps for several sizes (squares $100 \times 100$ and $225 \times 225)$ and for several initial distances $(\psi(0)=0.01$ and $\psi(0)=0.0025)$. The full curve represents the quenched model for $\psi(0)=0.5$. We see that the annealed and the quenched model have different phase transitions with $p_{\mathrm{c}}^{\text {annealed }}=0.22$ from directed percolation [6] and $p_{c}^{\text {quenched }} \sim 0.26 \pm 0.02$ from these data.

This discontinuity in the quenched square lattice is reminiscent of other phase transitions, like the ferromagnetic Curie point. If we identify $\psi(0)$ with the magnetic field $H$ and $\psi(\infty)$ with the magnetization $M$, then for the paramagnet, $p<p_{c}$, the magnetization $\psi(\infty)$ goes to zero if the field $\psi(0)$ goes to zero, with a constant «susceptibility" $\psi(\infty) / \psi(0)$ for small fields. On the ferromagnetic side, $p>p_{\mathrm{c}}$, we have on the other hand a finite spontaneous magnetization $\psi(\infty)$, if the field $\psi(0)$ approaches zero. The case $\psi(0)=0$ is pathological for both the Kauffmann model and the ferromagnet. Indeed, just as the susceptibility in the paramagnet, according to fig. 3 also our ratio $\psi(\infty) / \psi(0)$ seems to diverge if $p_{\mathrm{c}}$ is approached from below, as do the sample-to-sample fluctuations of $\psi(\infty)$ in one dimension, for the quenched model we never expect to find the chaotic phase, even by increasing the number of neighbours; we confirmed this expectation numerically with $K=2$ and $K=4$.

ATLAN et al. [2] studied similarly the influence of a small disturbance $\biguplus(0)$, called "noise", on the limit cycles, and found it sometimes to increase, sometimes not. If we simply investigate $\psi(t) / \psi(0)$ for intermediate times, we find it to increase drastically with $t$ for $p$ above $p_{\mathrm{c}}$ and to remain roughly constant for $p$ below $p_{\mathrm{c}}$.

In this work we have seen that Kauffman's model exhibits a phase transition in all dimensions $d \geqslant 1$ in its annealed version and in dimension $d=2$ for its quenched version. From fig. 2 we estimate

$$
p_{\mathrm{c}}^{\text {quenched }}=0.26 \pm 0.02 .
$$

We think that the quenched model has a phase transition for $d>1$, but not for $d=1$. The criteria we have used to see the phase transition are based on the history dependence of the asymptotic overlap, see eq. (10). 


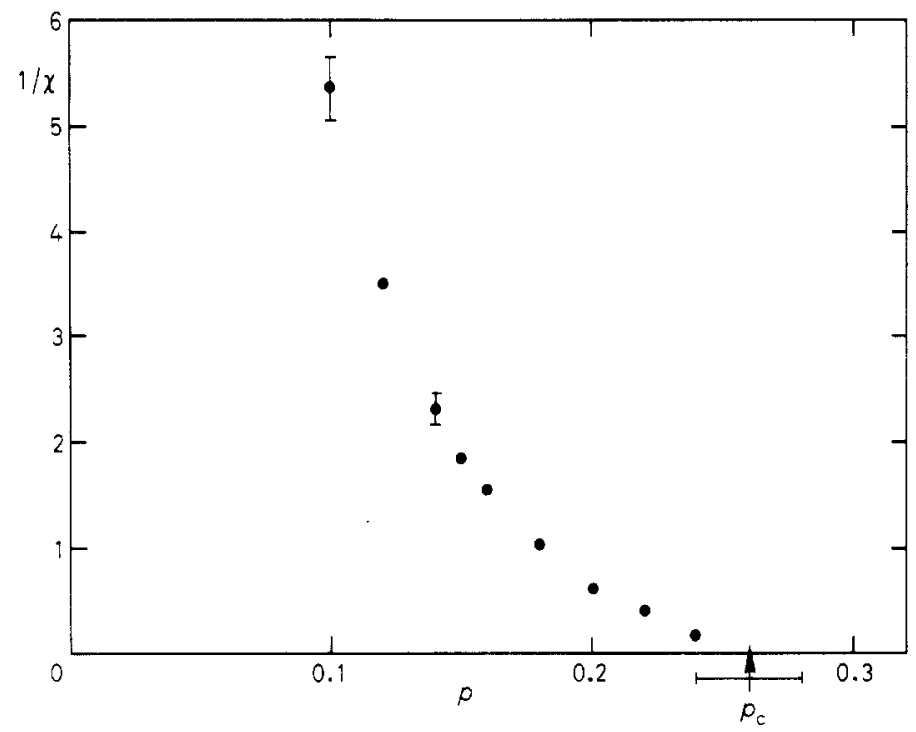

Fig. 3. - Susceptibility $\chi$ defined by (10) vs. $p$ for the quenched model in the frozen phase. $\chi$ was computed as $\psi(\infty) / \psi(0)$ for $\psi(0)=0.005$ and $\psi(0)=0.0025$. The susceptibility diverges at $p_{\mathrm{c}} \sim 0.26 \pm 0.02$.

It would be interesting to look for more accurate measurements of the transition threshold $p_{c}$ and for the critical exponents in the quenched case. Figure 2 shows, however, that $p_{\mathrm{c}}$ for the annealed model is smaller than for the quenched model on the square lattice.

It would also be interesting to look with high precision at other properties proposed in the past to characterize a phase transition, like the response to disturbances during the limit cycle [2], the dependence of the period length on system size and $p$, the percolation of «forcing structures» $[2,4,7]$ or of the network of frozen-in spins $[4,7]$, and to see whether these quantities exhibit a phase transition at the same threshold.

$$
* * *
$$

We thank G. WEISBUCH for helpful discussions.

\section{REFERENCES}

[1] Proceedings of the Los Alamos Conference, in Physica D, 10 (1984).

[2] Kauffman S. A., J. Theor. Biol., 22 (1969) 437; Physica D, 10 (1984) 145; and in Disordered Systems and Biological Organisation, edited by BIENENSTOCK E., FogelmaN Soulié F. and Weisbuch G. (Springer Verlag, Heidelberg) 1986, p. 339; Atlan H., Fogelman Soulié F., Salomon J. and Weisbuch G., Cybernetics and Systems, 12 (1981) 103; Gel'Fand A. E. and Walker C. C., Ensemble Modelling (Marcel Dekker, New York, N. Y.) 1984.

[3] Derrida B. and Pomeau Y., Europhys. Lett., 1 (1986) 45; Derrida B. and Weisbuch G., J. Phys. (Paris), to be published.

[4] Fogelman Soulié F., Thesis, Grenoble University (1985).

[5] Kinzel W., Z. Phys., 58 (1985) 229; Grinstein G., Jayaprakash C. and Yu He, Phys. Rev. Lett., 55 (1985) 2527; NoEST A. J., preprint (1986); ViChNIAC G. Y., TAMAYo P. and HARTMAN H., preprint (1986).

[6] Adler J., Deutscher G. and Zallen R. (Eds.), Percolation Structure and Processes, in Ann. Israel Phys. Soc., Vol. 5 (Adam Hilger, Bristol) 1983; in particular reviews of W. KINZEL and S. Redner; Domany E. and Kinzel W., Phys. Rev. Lett., 53 (1984) 311.

[7] Weisbuch G. and Stauffer D., preprint. 